Classification

Physics Abstracts

$61.16 \mathrm{D}-81.40 \mathrm{P}-71.45 \mathrm{G}$

\title{
High resolution electron spectroscopic imaging (ESI) of reverse micelles
}

\author{
Jean Michel Martin, Jean-Louis Mansot, Martine Hallouis and Hubert Tenailleau \\ Laboratoire de Technologie des Surfaces, URA CNRS 855, Ecole Centrale de Lyon, B.P. 163, 69131, \\ Ecully Cedex, France
}

\begin{abstract}
Résumé. - Nous avons examiné des micelles inverses de sulfonate de calcium surbasées par Microscopie Electronique filtrée en énergie. Lataill des coeurs est comprise entre 2 et $3 \mathrm{~nm}$. Les nicelles sont préalablement déposées sur une lame mince très fine de carbone amorphe. Nous avons obtenu des spectres EELS et des images filtrées en énergie, lesquelles son interprétées en considérant l'existence de quatre types de contraste : le contraste dû à la diffraction des atomes de calcium, le contraste de phase du support granuleux de carbone et de la forme sphérique de la micelle, le contraste spectroscopique dû à l'excitation des niveaux électroniques, et en particulier l'ionisation des niveaux de coeurs. En choisissant le mode de fonctionnement du microscope, chaque contraste peut être étudié en détail. Nous montrons que les images filtrées obtenues à partir des pertes faibles permettent de résoudre le coeur des micelles riche en calcium (diamètre $2-3 \mathrm{~nm}$ ) et la couche de molécule organique l'entourant (épaisseur 1-2 nm). L'image spécifique de cette couche est attribuée à un effet d'épaisseur massique modulé par la diffraction des atomes de calcium du coeur.
\end{abstract}

\begin{abstract}
Overbased calcium alkylaryl sulfonate reverse micelles of core diameter 2-3 nm are investigated by means of an Energy-Filtering Electron Microscope (EFEM). For this purpose, micelles are deposited on a very thin carbon film. Both selected area EELS spectra energy loss images are obtained and discussed. Data are interpreted considering the existence of mainly four contrasts : contrast caused by elastic scattering at calcium nuclei, phase contrast due to the grainy support and the micellar shape, spectroscopic contrast due to plasmon losses in carbonaceous materials and elemental chemical contrast due to the excitation of atomic electrons, and particularly the ionization of inner-shell electrons. Depending on the operating mode of the microscope, each contrast can be emphasized and studied. The main result is that Electron Spectroscopic Imaging (ESI) with low energy losses, permits high resolution images of the calcium-rich core (2-3 nm diameter) and of the organic shell (1-2 nm thick). The specific image of the organic shell is attributed to a mass-thickness effect modulated by elastic scattering at calcium atoms. This gives a definite picture of the composite structure of overbased reverse micelles.
\end{abstract}

\section{Introduction.}

The determination of the structure of reverse micellar systems is a subject of intense research, with regard to the very wide application fields of these compounds (catalysis, detergency, lubrication, 
etc...).

Overbased calcium alkylaryl sulfonate micelles are widely used as anti-corrosive additives in lubricants, and especially in motor oils. Such systems generally consist of an amorphous mineral core (2-3 nm diameter) surrounded by a shell made up amphiphilic molecules which prevent the core from floculation in hydrocarbon media.

In this case, the mineral core consists of amorphous calcium compounds such as carbonate and hydroxide, which diameter lies in the $2-3 \mathrm{~nm}$ range. The microstructure of the core has already been studied by electron diffraction and more accurately by Extended X-ray Absorption Fine Structure (EXAFS) at the calcium K-edge [1]. The morphology of such systems can be determined by light scattering [2], small angle X-ray scattering [3] and neutron scattering experiments [4]. Generally, the data obtained are in good agreement with the structural model proposed.

Conventional Transmission Electron Microscopy (CTEM) can be used to study such systems out of their equilibrium conditions because overbased micelles can be very easily isolated on a thin carbon film [5], without undergoing any visible structural transformation. On the other hand, frozen thin liquid film of micelles are also observable in the CTEM, due to the recent progress in cryotechnology applied to electron microscopy $[6,7]$.

With regard to the application of CTEM to the study of reverse micelles, the main advantage is the rapidity of the preparation, but several difficulties are immediately to be pointed out :

- the scattering contrast is very low, so that micelles are difficult to image,

- the observation of very small particles by phase contrast can be mixed up with the phase contrast of the carbon support,

- there is no analytical data concerning the elementary composition.

In this work, we used a Transmission Electron Microscope (TEM) equipped with a CastaingHenry energy filter (ZEISS CEM902), to study overbased reverse micelles of 2-3 nm diameter core. The aim of this work is to show the advantage of Energy Filtering Electron Microscopy (EFEM) to obtain electron images of the different components of the micelle. The interpretation of electron images is discussed and new perspectives are opened out.

\section{Preparation of specimens.}

Overbased reverse micelles consisted of calcium rich amorphous mineral cores $(2-3 \mathrm{~nm}$ diameter) surrounded by a shell of didodecyl benzene sulfonate molecules (theoretical length of the molecule $\approx 1.7 \mathrm{~nm}$ ). For this study, the system was purified by centrifugation and dialysis in hexane through an elastomer membrane in order to eliminate solid particles, organic impurities and high molecular weight hydrocarbons. The remaining colorless dispersion was used for the EFEM study. One drop of the highly diluted micellar dispersion in hexane was deposited onto a very thin carbon film mounted on a copper grid. After evaporation of the solvent, micelles sticked on the carbon film by Van der Waals interactions. For these experiments, special attention was paid to the thickness of the carbon support film which is used, in any case it must be inferior to $5 \mathrm{~nm}$ for good imaging conditions.

\section{A brief overview of EFEM.}

For the whole study, inelastic imaging was systematically performed after regarding EELS spectra on the specimen, in order to have a better understanding of the resulting contrasts obtained in the inelastic mode.

The ZEISS microscope was operating at $80 \mathrm{kV}$ accelerating voltage, the maximum scattering angle was limited to $17 \mathrm{mrad}$ by the $90 \mu \mathrm{m}$ diameter objective aperture used. The filter lens was 
operating in the diffraction mode, the spectrometer entrance aperture acting in this case as a selecting area aperture. The EELS spectra were recorded in the spectrum mode [8], the energy resolution being limited by the elctron gun $\left(\mathrm{LaB}_{6}\right.$ filament $)$, the Boersch effect, the width of the energy slit in the back focal plane of the filter, the solid acceptance angle and specimen area selection [8]. It was measured as $1.5 \mathrm{eV}$ (FWHM) on the zero loss peak. EELS spectra were sequentially recorded by means of a photomultiplier detector.

For ESI with the energy-selecting filter, we used a $1 \mathrm{~mm}$ entrance aperture, and adjustable slit was introduced in the back focal plane of the filter (EELS spectrum) and the energy filtered images were directly displayed, using projector lenses, on the TEM screen. The chromatic aberration depends on the energy window, typically calculation shows that the chromatic aberration disk diameter (in Angströms) is of the same order than the energy window (in electron-volts). Due to the second order aberration figure of the filter, there is an energy dispersion in the final image, typically the dispersion is about $10 \mathrm{eV}$ from the center of the image to the edge of the $1 \mathrm{~mm}$ filter entrance aperture, visible on the negative plate. For inelastic imaging, we used a low light level camera coupled to an image processing unit (KONTRON).

For more details on the operation modes of the ZEISS 902 electron microscope, see reference [8].

\section{Results and discussion.}

With regard to our specimen (micelles deposited on a carbon film), the different contrasts which are expected in the final electron image are the following :

- phase contrast due to the granular structure of the carbon support and the spheroidal shape of the micelle (Fresnel fringe),

- scattering constrat due to electrons elastically scattered at atoms and especially calcium atoms of the amorphous mineral core,

- spectroscopic constrast due to electrons inelastically scattered by the specimen and mainly due to plasmon losses in carbonaceous material,

- elemental constrast due to the inelastic scattering at specimen atoms (inner-shell ionization of carbon and calcium atoms).

Note that both scattering and spectroscopic contrasts are mass-thickness dependent but that multiple inelastic scattering is negligible in our conditions.

Actually, the situation is quite complex, because it is not possible by angle and energy selection in the microscope, to isolate completely the different contributions. In any case, for example, both scattering and phase contrasts are convolved in the inelastic images, depending on the energy window which is chosen..

To obtain the maximum of information on our specimen, we used successively four ESI imaging mode available in the ZEISS 902 microscope :

(1) zero-loss bright field (BF),

(2) zero-loss annular dark field (ADF) (also called strioscopic dark field),

(3) inelastic bright field ( $\Delta E$-loss $\mathrm{BF})$ at a given energy loss $\Delta E$ and an energy window well apart from any absorption edge,

(4) elemental mapping, which is an inelastic BF obtained from a selected inner-shell ionization edge, characteristic of the elementary composition (with or without background correction). (Note that BF and ADF images are here referred to the objective lens image formation only)..

In table $I$, an attempt is made to show how the different contrasts are qualitatively distributed in each Electron Spectroscopic Image (with regard to our specimen configuration). In any case, 
Table I. - Qualitative representation of the distribution of contrasts in different EFEM operating conditions (for our specimen configuration).

\begin{tabular}{|c|c|c|c|c|c|}
\hline & $\begin{array}{c}\text { Pure phase } \\
\text { contrast }\end{array}$ & $\begin{array}{c}\text { Scattering } \\
\text { contrast }\end{array}$ & $\begin{array}{c}\text { Spectroscopic } \\
\text { contrast }\end{array}$ & $\begin{array}{c}\text { Elemental } \\
\text { contrast }\end{array}$ & $\begin{array}{c}\text { Relative } \\
\text { signal } \\
\text { intensity }\end{array}$ \\
\hline $\begin{array}{c}\text { Zero loss } \\
\text { BF }\end{array}$ & strong & weak & no & no & 1 \\
\hline $\begin{array}{c}\text { Zero loss } \\
\text { ADF }\end{array}$ & no & strong & no & no & $\approx 10^{-2}$ \\
\hline $\begin{array}{c}\text { inelastic } \\
\Delta E-\text { loss BF }\end{array}$ & weak(1) & strong & strong & no(2) & $\approx 10^{-3}$ \\
\hline $\begin{array}{c}\text { Elemental } \\
\text { mapping }\end{array}$ & no(3) & strong & no(4) & strong & $\approx 10^{-4}$ \\
\hline
\end{tabular}

(1) mainly limited by the energy window (chromatic aberration).

(2) if the energy window is located well apart from an inner-shell ionization edge.

(3) generally not observed.

(4) when using a background correction.

the electron signal in the zero-loss BF represents more than $95 \%$ of the total signal penetrating through the specimen (thickness inferior to $10 \mathrm{~nm}$ ).

Apart from the case of zero-loss images, which do not contain any elemental information, all kind of contrasts are more or less combined in each image ; but in some cases, the contribution is found negligible, or not visible with the operating conditions of the electron optics. For example, ADF images contain a strong contribution of scattering contrast, modulated by the thickness effect ; at the opposite, inelastic BF images contain a major contribution of mass-thickness contrast due to plasmon losses, modulated by scattering and phase contrasts. Elemental mapping show a strong chemical contrast, but also modulated by elastic scattering, to the same extent that any other inelastic image (neglecting channeling effects in crystalline materials). Phase contrast, if not desirable, can be reduced when working at focus of the objective lens.

4.1 ZERO-LOSS ESI OF MICELLES - A zero-loss bright field (BF) image of micelles is shown in figure 1a. The energy window was $10 \mathrm{eV}$ wide, centered on the zero-loss peak. The image was obtained at focus (original magnification X85000). The calcium-rich core ( $3 \mathrm{~nm}$ diameter) is imaged; due to the tiny contrast in the zero-loss BF image, the organic shell, which is present between contacting micelles, cannot be observed. (Note that when slightly defocussing, phase contrast from the carbon support is transferred into the final image, to the extent that micelles are practically no visible in these conditions.

A zero-loss annular dark field (ADF) image of micelles is presented in figure 1b. The enhanced scattering contrast of calcium atoms makes that the mineral cores are resolved, unfortunately the diffraction of very small carbon crystallites from the support is also transferred in the ADF image as a noisy background. As expected from table I, zero-loss images are dominated by elastic scattering of calcium cores and no information is available from the organic shcll contribution. 

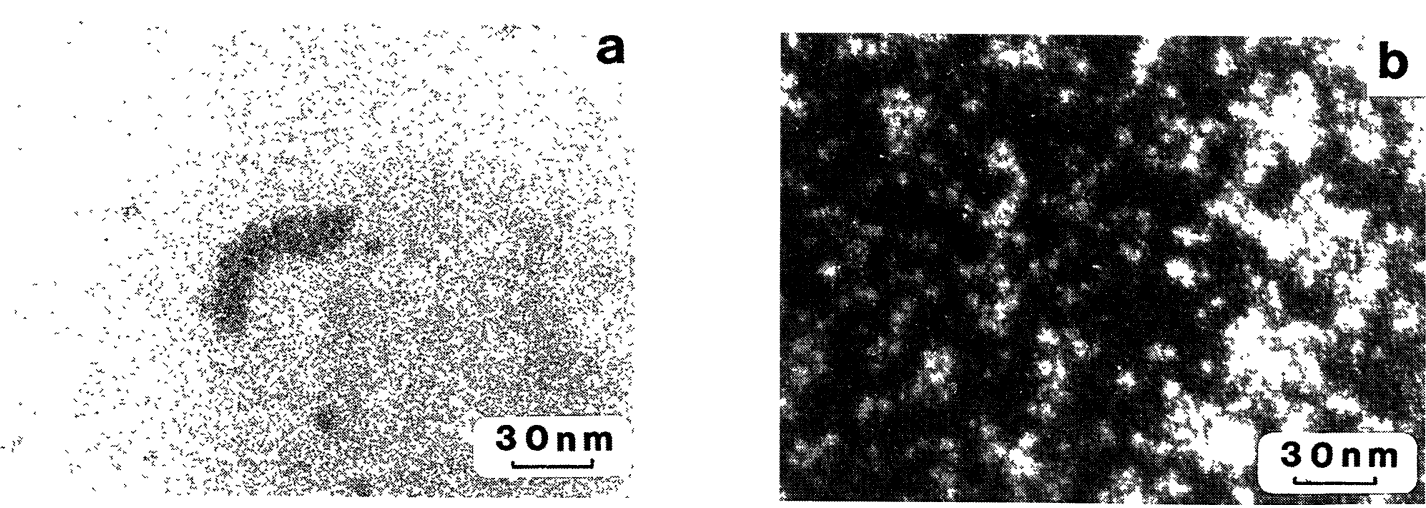

Fig. 1. - Electron Spectroscopic Imaging (ESI) of reverse micelles. a) Zero-loss bright field (BF). b) Zeroloss annular Dark Field (ADF).

4.2 LOW-ENERGY ESI OF MICELLES - Considering our material, the low-loss EELS spectrum shows two main features (Fig. 2a) : the plasmon loss excitation of carbonaceous species (maximum near $25 \mathrm{eV}$ ) and the calcium $M_{2.3}$ core loss excitation beginning at $36 \mathrm{eV}$, which is relatively well defined.

When imaging at low energy-losses in the ZEISS 902 microscope, one must take into account the energy dispersion in the final image due to the second-order aberration which is about $2 \mathrm{eV} / \mathrm{cm}$ on the negative plate. This can be advantageously used here to study, in detail, the evolution of the filtered image of very small specimens over a few eV energy loss. For example, figure $2 \mathrm{~b}$ shows a low loss image of micelles when the energy window of $5 \mathrm{eV}$ width is centered at $36 \mathrm{eV}$ loss in the back focal plane of the filter ( $\mathrm{Ca} M_{2.3}$ core loss excitation energy). On the same electron micrograph, an advantage is that the spectroscopic imaging of micelles is continuously visible from $25 \mathrm{eV}$ (the edge of the negative) to $36 \mathrm{eV}$ (the center of the negative). As the original magnification was X85000, the difference of focus between the two imaging conditions is not visible and it is readily seen the important change and the contrast reverse bctween the two situations : at $36 \mathrm{eV}$ loss ( $\mathrm{Ca} M_{2.3}$ edges) the core of the micelle is imaged, at $26 \mathrm{eV}$ loss (carbon plasmon) the shell is specifically imaged.

Figure 3a shows an inelastic image taken at $\Delta E=26 \mathrm{eV}$ (in the carbon plasmon-loss region) in the same conditions ; the organic shell of the micelle is imaged (see enlargment in Fig. $3 b$ ) the thickness can be estimated between 1-2 nm, in good agreement with the theoretical length of the surfactant molecule $(1.7 \mathrm{~nm})$. Figures $3 \mathrm{c}$, 3d shows an inelastic image of the same area, at $36 \mathrm{eV}$ loss ; as mentioned earlier, even without any background correction, the core of the micelle (diameter $1-3 \mathrm{~nm}$ ) is now clearly imaged. The light zones in the images pinpoint the atoms at which the $\Delta E$ energy loss electrons were scattered.

The plasmon loss image is very interesting because only a portion of surfactant molecules are imaged. It is unlikely that the signal comes from a selective plasmon excitation in the organic shell only ( $2 \mathrm{~nm}$ resolved) due to the delocalization of this interaction. The $130 \mathrm{eV}$ loss BF image (Fig. 4) shows the same contrast, although that the signal is here very low. This confirms definitely that this shell contrast is not related to any selective interface plasmon excitation. On the other hand, a surface plasmon excitation is also excluded in this energy band.

Consequently the inelastic image generally shows a mass thickness (volume plasmon) contrast with a high resolution power. 
a
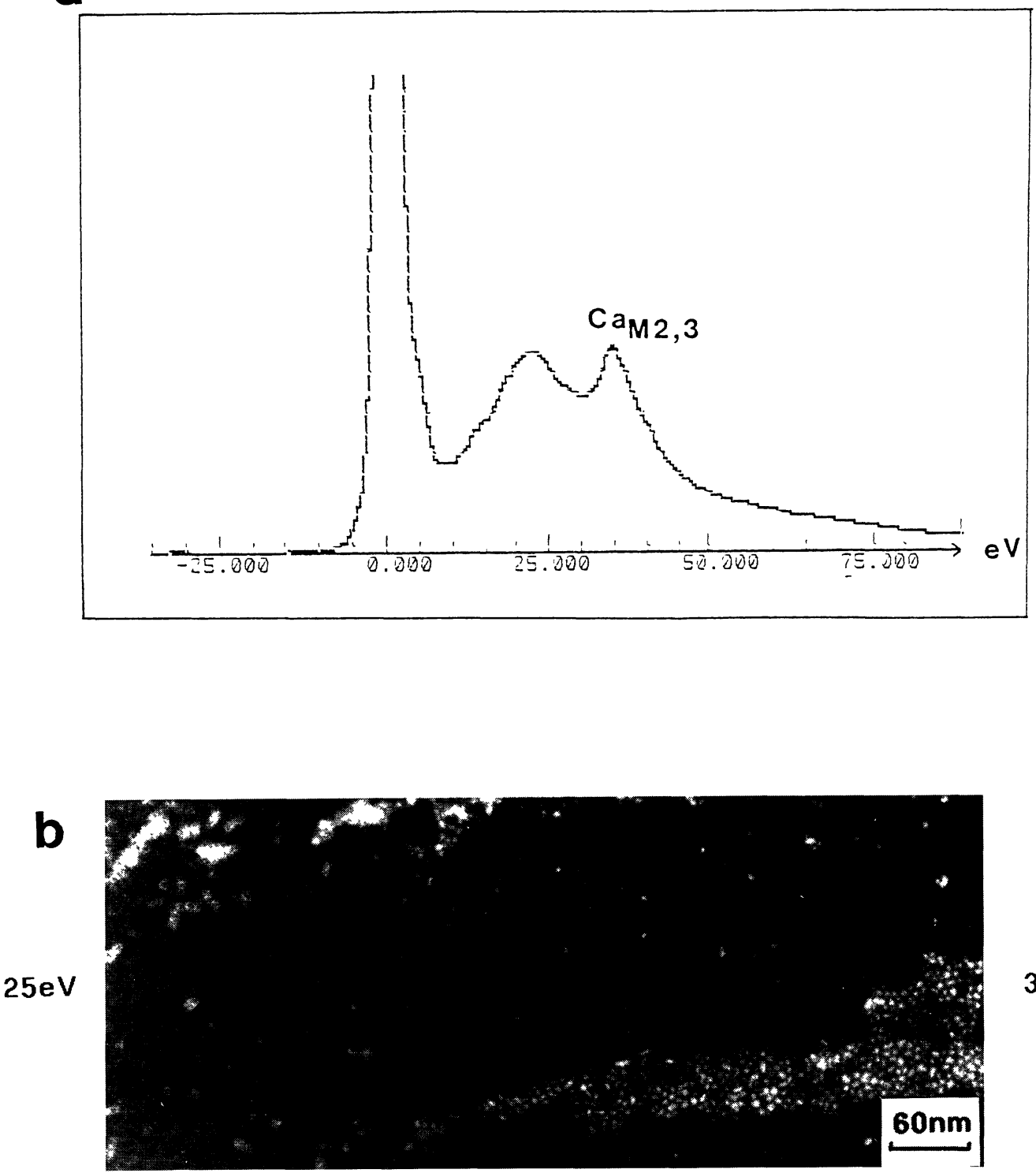

$36 \mathrm{eV}$

Fig. 2. - a) Low energy-loss EELS spectrum of micelles. b) Low energy-loss image of reverse micelles. Note the evolution of contrast when the energy dispersion in the image varies continuously from $25 \mathrm{eV}$ loss to $36 \mathrm{eV}$ loss.

As phase contrast is transferred to the image, scattering contrast due to the objective aperture is also present in inelastic electrons. Therefore, both the $26 \mathrm{eV}$ and $130 \mathrm{eV}$ loss $\mathrm{BF}$ images can be interpreted as a high resolution mass-thickness contrast modulated by the clastic scattering at calcium atoms in the micelle core. Fortunately, the result is a definite picture of the location of surfactant molecule around the calcium rich core. 

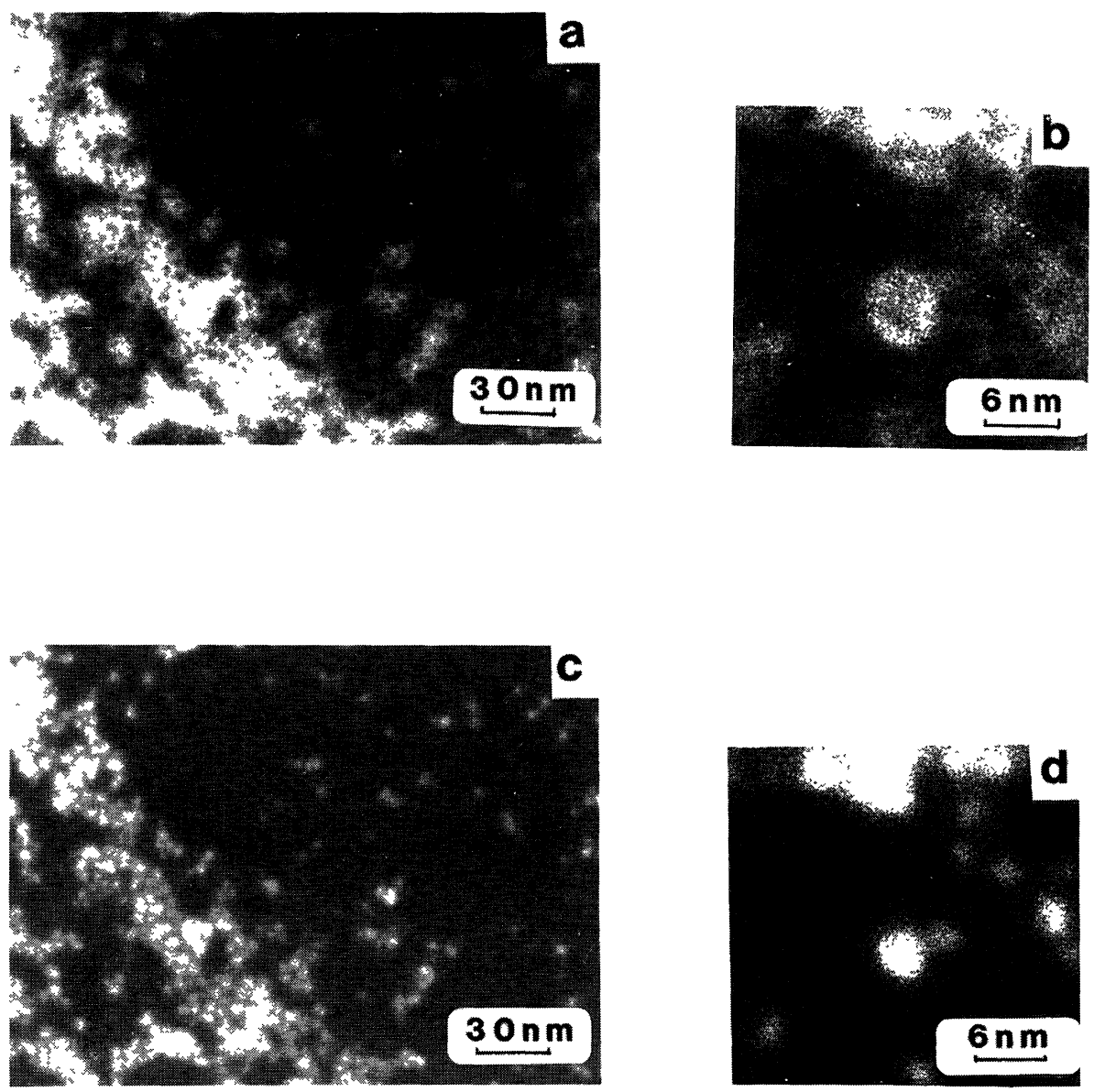

Fig. 3. - Electron Spectroscopic Imaging (ESI) of reverse micelles. a) $\Delta E=22 \mathrm{eV}$ (carbon plasmon loss region), c) $\Delta E=36 \mathrm{eV}$ ( $\mathrm{Ca} M_{2.3}$ edge) The enlargments (b) et (d) show complementary images for one reverse micelle.

Such spatial resolution is not routinely attainable with the conventional STEM mapping process with the EDX detector. Concerning the chemical mapping of atoms by the EELS technique, using inner-shell ionization edges and suitable background correction techniques, the atomic resolution (inferior to $1 \mathrm{~nm}$ ) has been demonstrated in ref. [9] using uranium atoms deposited on a carbon film. Our data suggest that high resolution elemental images can also be obtained, in certain cases, by combining plasmon loss and scattering contrasts.

Apart from the micelle, filtered images also contain some contrasts from the carbon support, a careful examination shows (Fig. 3a, b) that this contrast does not depend on the encrgy loss. Consequently, this contrast is attributed either to phase contrast or to local mass-thickness variations, which are transferred into the inelastic signal, in agreement with theoretical prediction [10]. Nevertheless, the phase contrast of carbon is only visible if the chromatic aberration disk is reduced, i.e. using a very narrow energy band (typically inferior to $5 \mathrm{eV}$ in our case). 


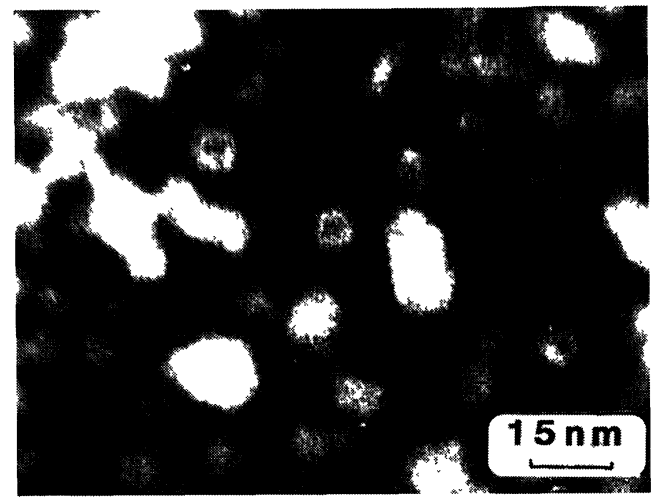

Fig. 4. - Electron Spectroscopic Imaging (ESI) of reverse micelles. $\Delta E=130 \mathrm{eV}$.

4.3 CORE LOSS ESI OF MICELLES - The EELS spectrum of carbon-depositcd micelles, obtained in the range $250-600 \mathrm{eV}$ is shown in figure 5 . The carbon $\mathrm{K}$-edge at $284 \mathrm{eV}$, the calcium sharp $L_{2.3}$ edges near $347 \mathrm{eV}$ and the oxygen K-edge at $532 \mathrm{eV}$ are displayed in this spectrum.

The carbon K-edge presents three fine structures which have already been recognized and discussed in a previous work [5] namely :

- the electronic transition from $1 \mathrm{~s}$ level to antibonding $\pi_{\mathrm{g}}^{*}$ molecular orbital of $\mathrm{sp}_{2}$ hybridizedcarbon in a C-C bonding (peaking at $285 \mathrm{eV}$ ),

- the electronic transition from $1 \mathrm{~s}$ level to $\pi_{\mathrm{g}}^{*}$ of $\mathrm{sp}_{2}$-hybridized-carbon in C-O bonding (peaking at $288 \mathrm{eV}$ loss),

- the electronic transition from 1 s level to $\sigma_{u}^{*}$ due to the existence of $s_{3}$-hybridized-carbon.

Of course, these transitions are characteristics of our material which respectively contains :

- $(\mathrm{C}=\mathrm{C}) \mathrm{sp}_{2}$ hybridized carbon both in the pseudographitic carbon of the support and in the ABS detergent molecule,

- $(\mathrm{C}=\mathrm{O}) \mathrm{sp}_{2}$ hybridized carbon in coplanar $\mathrm{CO}_{3}$ - - anions (calcium carbonate),

- (C-C) $\mathrm{sp}_{3}$ hybridized carbon from the hydrophobic part of the detergent molecule.

The calcium K-edge presents two sharp peaks (white lines corresponding to $L_{2.3}$ edges $\left(2 \mathrm{p}_{1 / 2}\right.$; $2 \mathrm{p}_{3 / 2} \rightarrow 3 \mathrm{~d}$ transitions).

The oxygen K-edge also displays some fine structures which are not well known at the moment. With regard to calcium, taking into account that the jump ratio is sufficiently high, and that the presence of the carbon K-edge does not permit a complete background correction for imaging, we used a simple two-images technique to obtain the net calcium mapping in our specimen (Fig. 6a, b, c, d). A selecting slit of $10 \mathrm{eV}$ guaranties a chromatic aberration disk inferior to $10 \AA$, two images were recorded with the video camera (dwell time $10 \mathrm{~s}$ ), first at $340 \pm 5 \mathrm{eV}$ (a) and $355 \pm 5 \mathrm{eV}$ (b), the substraction (b)-(a)) gives the calcium map distribution (c) : figure $6 d$ represents figure $6 c$ which as been processed (high-pass filter and smoothing). As can be seen, the calcium rich core of the micelle is imaged, and the resolution is good enough to distinguish the detergent layer between contacting particles $(2-3 \mathrm{~nm})$.

\section{Conclusion.}

Overbased calcium alkylaryl sulfonate micelles (2-3 $\mathrm{nm}$ core diameter) deposited on a thin carbon film were studied by Energy-Filtering Electron Microscopy (EFEM) at $80 \mathrm{kV}$ by means of 

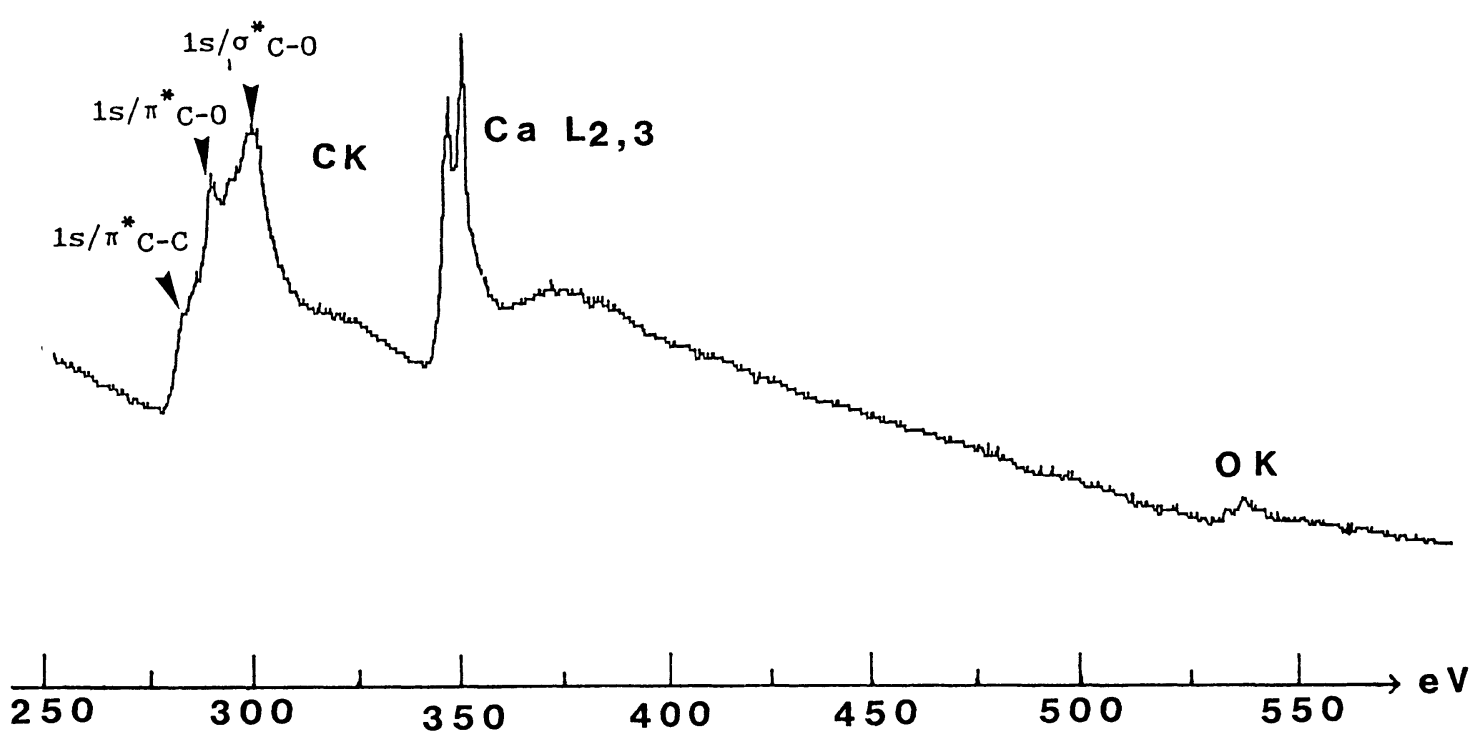

Fig. 5. - Core loss EELS spectrum of carbon-deposited micelles showing inner-shell ionization edges.

Electron Spectroscopic Imaging (ESI).

Main results are the following:

- low energy-loss ESI of reverse micelles allows high resolution images to be obtained of both the calcium rich core (2-3 nm diameter) and the surfactant molecule shell (1-2 nm length). The calcium ESI image can be easily interpretated as an elemental mapping from the $\mathrm{Ca} M_{23}$ ionization edge beginning at $36 \mathrm{eV}$ loss (without any background correction). The organic shell specific image can be obtained from the carbon plasmon loss region (between 10 and $36 \mathrm{cV}$ ) but also from higher energy losses (between 100 and $200 \mathrm{eV}$ ) of the EEL spectrum. The molccule ESI image is attributed to a mass thickness effect in carbonaceous material modulated by clastic scattering at calcium nuclei. This pseudo-image of the organic shell must not be confused with a surface or interface plasmon loss image due to electrons not entering the micelle material. It is also important to note that these images are obtained with a small energy-selecting aperture, allowing phase contrast to be present in all ESI images,

- core energy-loss ESI of micelles were obtained from the $\mathrm{Ca} L_{23}$ edge at $347 \mathrm{eV}$ loss. The elemental mapping of calcium (with a two-images correction background) is in good agreement with the $\mathrm{Ca}_{23}$ edge specific image, and confirms the location of calcium atoms in the micelle. The analysis and assignment of fine structures in the carbon K-edge of the EEL spectrum are in good agreement with the expected molecular structure of the surfactant molecule giving another analytical support of the existence of the organic shell.

Next EFEM investigations using cryo fixed micelles thin films are currently under consideration. 


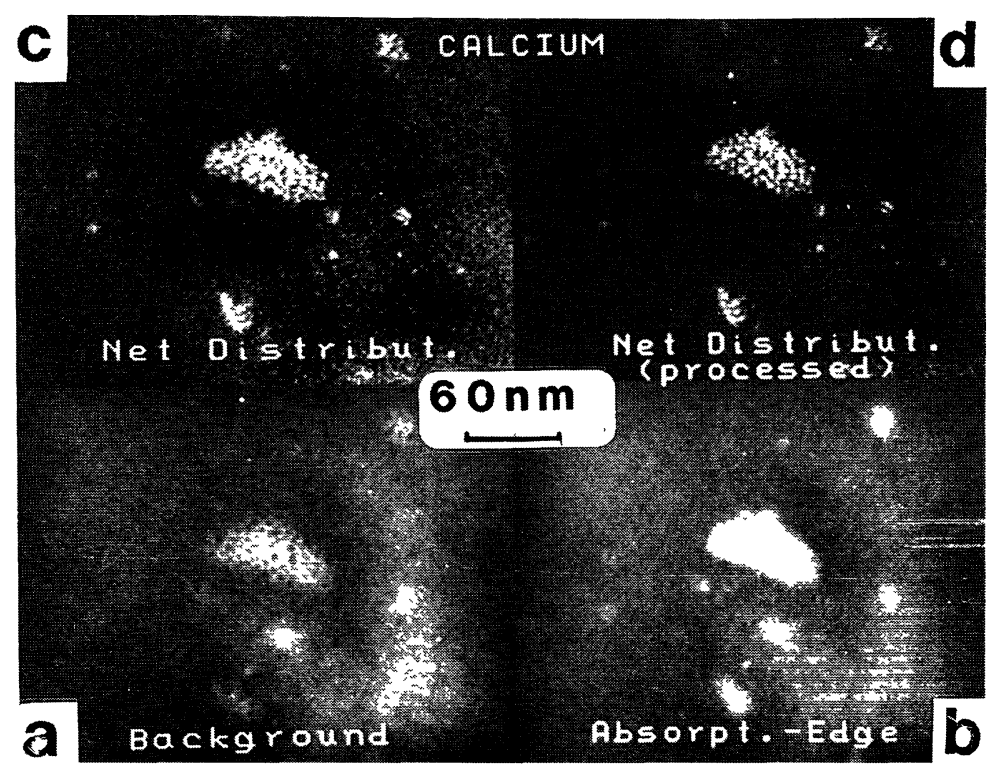

Fig. 6. - Electron Spectroscopic Imaging (ESI) of reverse micelles. a) background, $\Delta E=340 \mathrm{eV}, \mathrm{b}$ ) $\mathrm{Ca} L_{2.3}$ edge, $\Delta E=355 \mathrm{eV}$, c) net calcium mapping, d) net calcium mapping (processed).

\section{Acknowledgements.}

The authors thank ELF Company for financial support and D. Faure and P. Hoornacrt for their helpful comments and for providing reference compounds. The authors thank also Dr. R. Bauer from Zeiss Company.

\section{References}

[1] Martin J.M., Belin M. and Mansot J.L., J. Phys. France 47 (1986) 887.

[2] HEILWEIL I.J., J. Colloid Sci. 19 (1964) 105-112.

[3] Glavaty O.L., Marchenko A.I., KravchoOK G.G. and Glavaty E.V., Acta Chim. Hungarica 116 (4) (1984) 367-375.

[4] MARKOVIC I. and OTTEWILl R.H., Colloid Polymer Sci. Part. II 264 (1986) 265-276.

[5] MARTIN J.M., MANSOT J.L. and HALlOUIS M., Ultramicroscopy 30 (1989) 321-328.

[6] WATKInS R.C., Phys. Technol. 15 (1984).

[7] VINSON P.K., Proceeding of the 46th Annual Meeting of the EMSA (1988), pp. 112-113.

[8] REIMER L., FROMM I. and RENNEKAMP R., Ultramicroscopy 24 (1988) 339-354.

[9] MORY C. and COLLIEX C., Ultramicroscopy 28 (1989) 339.

[10] WANG Z.L., Proc. Proceeding of the 46th Annual Meeting of the EMSA (1988), pp. 818-819.

Cet article a été imprimé avec le Macro Package "Editions de Physique Avril 1990". 